\title{
Selective Hemorrhoidal Dearterialization with High Mucopexy in the Surgical Treatment of Hemorrhoidal Disease
}

\author{
Carlos Mateus Rotta10 Stephanie Drago ${ }^{2(1)}$ Afonso Henrique da Silva e Sousa $\mathrm{Jr}^{3(1)}$ \\ Carlos Augusto Real Martinez ${ }^{4,5(-)}$ Marjorie Cristina da Cruz Bernardino ${ }^{6(0)}$ \\ ${ }^{1}$ Departament of Coloproctology, Faculty of Medicine, Universidade \\ de Mogi das Cruzes, Mogi das Cruzes, SP, Brazil \\ 2 Medical school, Universidade de Mogi das Cruzes, Mogi das Cruzes, \\ SP, Brazil \\ ${ }^{3}$ Department of Surgery, Universidade de São Paulo, São Paulo, SP, Brazil \\ ${ }^{4}$ Graduate Studies in Health Sciences, Universidade de São Francisco, \\ Bragança Paulista, SP, Brazil \\ Address for correspondence Carlos Mateus Rotta, Rua Júlio Prestes, \\ 240, Mogi das Cruzes, SP, CEP 08780-110, Brasil \\ (e-mail: Mateusrotta@saluspraxis.com). \\ ${ }^{5}$ Department of Surgery, Faculty of Medical Sciences, Universidade \\ Estadual de Campinas, Campinas, SP, Brazil \\ ${ }^{6}$ Statistics, Universidade de Mogi das Cruzes, Mogi das Cruzes, SP, Brazil
}

J Coloproctol 2021;41(3):234-241.

\author{
Abstract \\ Keywords \\ - hemorrhoids \\ - hemorrhoids/surgery \\ - hemorrhoidectomy \\ - ligation \\ - ultrasonography \\ - doppler \\ - pulsed \\ - transanal endoscopic \\ surgery
}

The doppler-guided transanal hemorrhoidal dearterialization technique associated with mucopexy is a noninvasive surgical option used to treat hemorrhoidal disease (HD).

Objective To compare and analyze the results using a variation of the doppler-guided transanal hemorrhoidal dearterialization technique with the technique of selective hemorrhoidal dearterialization with high mucopexy in the treatment of HD.

Method A total of 292 patients who underwent surgical treatment for grade II, III and IV HD from March 2012 to December 2017 were studied. From this total, 110 (37.6\%) patients underwent a conventional doppler-guided transanal hemorrhoidal dearterialization with mucopexy (CD), and 182 (62.3\%) underwent selective hemorrhoidal dearterialization with high mucopexy (SHeLF). In the group of patients undergoing CD, 4 patients (3.64\%) had grade II HD, $82(74.55 \%)$ grade III, and $24(21.82 \%)$ grade IV. In the group submitted to SHeLF, 18 (9.89\%) patients had grade II HD, $86(47.25 \%)$ had grade III, and 65 (35.71\%) had grade IV. The same surgeon operated all patients under spinal anesthesia. In patients undergoing $C D$, six arterial branches have been dearterialized, while in patients undergoing SHeLF, the hemorrhoidary nipples submitted to a dearterialization were selected (from 1 to 5 ) by intraoperative evaluation followed by

Study performed at the Hospital das Clínicas Luzia de Pinho Melo, Mogi das Cruzes, SP, Brazil - Department of Surgery, Faculty of Medicine, Universidade de Mogi das Cruzes, Mogi das Cruzes, SP, Brazil.

received

January 5, 2021

accepted after revision

June 15, 2021
DOI https://doi.org/

$10.1055 / \mathrm{s}-0041-1735544$

ISSN 2237-9363. (c) 2021. Sociedade Brasileira de Coloproctologia. All rights reserved.

This is an open access article published by Thieme under the terms of the Creative Commons Attribution-NonDerivative-NonCommercial-License, permitting copying and reproduction so long as the original work is given appropriate credit. Contents may not be used for commercial purposes, or adapted, remixed, transformed or built upon. (https://creativecommons.org/ licenses/by-nc-nd/4.0/)

Thieme Revinter Publicações Ltda., Rua do Matoso 170, Rio de Janeiro, RJ, CEP 20270-135, Brazil 
high rectal mucopexy. In the postoperative period, the following parameters were evaluated: pain, tenesmus, bleeding, and recurrence.

Moderate results to severe pain was a postoperative complaint reported by $13(11.82 \%)$ patients undergoing $C D$, and by $19(10.44 \%)$ undergoing SHeLF. Intense tenesmus was reported by $26(23.64 \%)$ patients undergoing CD and by 7 (3.85\%) undergoing SHeLF. Three patients $(2.73 \%)$ undergoing CD and 1 (0.55\%) undergoing SHeLF evolved with postoperative bleeding. One patient $(0.55 \%)$ in the group undergoing $C D$ required surgical review of hemostasis. Six patients (5.45\%) who underwent CD and 8 (4.39\%) who underwent SHeLF were reoperated due to disease recurrence.

Conclusion Comparing statistics, patients undergoing the SHeLF technique have less postoperative pain, tenesmus and postoperative bleeding when compared with CD.

\section{Introduction}

Hemorrhoidal disease (HD) is a common disease; ${ }^{1}$ in industrialized countries, it is estimated that $50 \%$ of individuals $>50$ years old develop some symptoms of the disease throughout life. ${ }^{2,3}$ Some individuals temporarily benefit from conservative measures. However, in those in whom the disease is refractory to clinical treatment, surgical treatment is usually imposed. ${ }^{4,5}$

From the $20^{\text {th }}$ century onwards, excisional techniques for the surgical treatment of HD were the most used, either by the open method that provides for the healing of the surgical wound by second intention, or by the closed technique that recommended the primary closure of the bloody area by means of suture. ${ }^{6,7}$ Even today, despite the postoperative painful intensity, the good long-term results obtained by both techniques made them be considered the "gold standard" techniques for the treatment of HD. Excisional hemorrhoidectomy by open or closed methods still serve as a comparison for the evaluation of the results obtained with new procedures that have been conceived for HD treatment.

In 1998, with the objective of treating HD and reducing postoperative pain, the hemorrhoidectomy technique with stapling retopexy (AM) was proposed. ${ }^{8}$ This excisional technique aimed to treat HD by interrupting submucous blood flow to hemorrhoidal cushions, removing the most proximal portion of these and fixating the remaining mucosa as possible. ${ }^{8}$ However, although the technique significantly reduces postoperative pain over time, the possibility of developing severe complications related to the procedure was shown, as well as a considerable long-term recurrence rate. ${ }^{9,10}$

With a better understanding of the etiopathogenesis of HD, blood hyperflux to hemorrhoidal cushions and the degradation of the conjunctival structure of support of these cushions enabling mucous prolapse in the mid-1990s, a new surgical strategy was proposed for the treatment of HD. ${ }^{11}$ The authors proposed the dearterialization of the nourishing vessels of the hemorrhoidal cushion by the transanal route. The arterial branch was identified by dopplerflowmetry. They also proposed that hemorrhoidal prolapse be treated by craniocaudal mucopexy by making a manual suture
$(\mathrm{DGHD}+\mathrm{M})$. The technique of $\mathrm{DGHD}+\mathrm{M}$ has as rationale to treat the main mechanisms associated with the development of HD: arterial hyperflow and the degradation of the supporting tissues of hemorrhoidal cushions. This technique is a noninvasive surgical option, as it is not excisional, and has been used to treat the various degrees of HD. ${ }^{1,12}$ In the DGHD + M technique, six arterial branches identified by Doppler are dearterialized throughout the circumference of the mucosa of the distal rehest, and mucopexy is performed by means of a manual suture that begins in the highest portion of the cushion and ends just above the pectinline line. ${ }^{1,12}$ The ligation of six arterial branches made the surgery more laborious. With this, in many cases, dearterialization was performed in a place where, although a prolapsed hemorrhoidal cushion was not identified, it was possible to identify arterial blood flow.

To interrupt blood flow more selectively, we started to perform transanal hemorrhoidal dearterialization to interrupt arterial blood flow only from the arterial branches of the prolapsed cushions and with hyperflow identified by Doppler flowmetry. After completing selective dearterialization, mucopexy of the prolapsed cushions was performed in a higher position when compared with the classical technique. Starting mucopexy cranially with the same wire from the X of arterial ligation and tying the arterial ligation, and only after the pexia, similar to DGHD + M, was started. Although we have been familiar with the classical technique of DGHD $+\mathrm{M}$ since 2010, selective hemorrhoidal hight lifting (SHeLF) was conceived based on the need to propose a new technical option that reduced the high rates of tenesmus, as well as anal pain and bleeding that present a worrying incidence after the classical technique. ${ }^{12}$ We believe that the reduction of these indexes could perhaps be achieved if we performed high mucopexy only in the cushions where there is significant arterial flow, which increases the volume of the hemorrhoidal cushion and, consequently, the prolapse. Therefore, this new technical option could confer a greater selectivity to the treatment. To the best of our knowledge, to date, this surgical option has not yet been proposed. Thus, the aim of the present study is to present this technical variation for the surgical treatment of HD, which was used in a series of cases, and to compare it with the DGHD + M technique. 


\section{Casuistry and Method}

The present study was reviewed and approved by the Research Ethics Committee of the Universidade de Mogi das Cruzes (UMC, in the Portuguese acronym) and was registered on the Brazil Platform with the CAAE number 32503019.9.0000.5497

All patients underwent complete proctological examination before surgical indication, noting the characteristics of $\mathrm{HD}$ and any associated diseases. Patients $>50$ years old before the surgical procedure underwent colonoscopy for colorectal cancer screening. The surgical interventions were always performed electively and sequentially and by the same surgeon at the School Hospital of the School of Medicine of the UMC. Patients with stratified HD in grades II, III and IV according to the Goligher classification were included. ${ }^{13}$ Patients with evolving hemorrhoidal thrombosis, patients with other anorectal disorders associated with HD, with inflammatory bowel disease, and those with neoplasms of any origin were excluded. Patients using oral anticoagulants had their treatments discontinued 1 week before the procedure or replaced by low molecular weight heparin therapy, applied subcutaneously, which was suspended on the eve of the procedure and reintroduced 24 hours later. For each patient, the following postoperative variables were recorded: duration of the operation, bleeding, presence of anorectal pain, prolapse, tenesmus, and evolution of the patient with hemorrhoidal thrombosis.

A total of 292 patients were consecutively operated between March 2012 and December 2017. The patients were divided into two groups: group 1, DGHD-M, in which the classical technique of Doppler-guided hemorrhoidal dearterialization associated with traditional mucopexy was performed; and group 2, SHeLF, in which the selective hemorrhoidal dearterialization technique with high mucopexia was performed. Group 1 had 110 patients (37.7\%); 63 (57.27\%) female and $47(42.73 \%)$ male. In group 2, 182 patients (62.3\%) were allocated; 98 (53.8\%) female and 84 (46.2\%) male. The mean age of the patients was 47.3 years old (17 to 94 years old) as shown in - Table 1 .

In the group of patients submitted to DGHD $+\mathrm{M} ; 4(3,64 \%)$ had grade II HD, 82 (74.55\%) grade III, and 24 (21.82\%) grade IV. In the group submitted to SHeLF, 18 patients (9.89\%) had

Table 1 Characteristic of the studied sample

\begin{tabular}{|l|l|l|l|}
\hline \multirow{2}{*}{ Total patients } & & DGHD + M & SHeLF \\
\cline { 3 - 4 } & & n (\%) & No. (\%) \\
\hline \multirow{3}{*}{ Grade } & II & $410(37.7)$ & $182(62.3)$ \\
\cline { 2 - 4 } & III & $82(7.64)$ & $18(9.89)$ \\
\cline { 2 - 4 } & IV & $24(21.82)$ & $65(47.25)$ \\
\hline \multirow{2}{*}{ Gender } & Female & $63(57.27)$ & $98(53.85)$ \\
\cline { 2 - 4 } & Male & $47(42.73)$ & $84(46.15)$ \\
\hline
\end{tabular}

Abbreviations: DGHD + M: Doppler-Guided Hemorrhoidal Dearterialization + craniocaudal mucopexy by manual suture, SheLF, selective hemorrhoidal high lifting. grade II HD, 86 (47.25\%) grade III, and 65 (35.71\%) grade IV, as shown in - Table 1.

\section{Equipment employed}

The system used is composed of an ultrasound signal generation unit and interpretation by pulsed vascular flow Doppler effect. The transducer contains a crystal designed to capture the production of the ultrasonic wave reflected by the moving blood in the arterial branches. Thus, the signal obtained is transformed by the system into an audible and expandable sound wave. The identification and location of the arterial branches is facilitated by the design of the equipment and by the movements of the hands of the surgeon, allowing the correct juxtaposition of the transducer on the arterial branch of the rectal mucosa to be identified. However, to obtain the technically expected effect, the trajectory of the ultrasound beam should reach the various layers of the rectal wall, without air interposition between the transducer and the rectal mucosa. To minimize this difficulty, transparent ultrasound gel is used, and the tip of the transducer is lightly touched on the rectal mucosa, in search of the sound signal characteristic of the presence of the blood vessel (arteriola of blood supply of the hemorrhoidal cushion). The anoscope used in most procedures was the Endopex model AC002, supplied by Hexagon Industry and Trade Orthopedic Implants LTDA (Campinas, SP, Brazil) (-Figure 1), which contains a work area as longitudinal cleft partially closed by a rotating sliding piece with the function of extending the work area in the longitudinal direction, so as to allow good operation of the transducer and, later, the suture of the vascular cushion to perform mucopexy in the

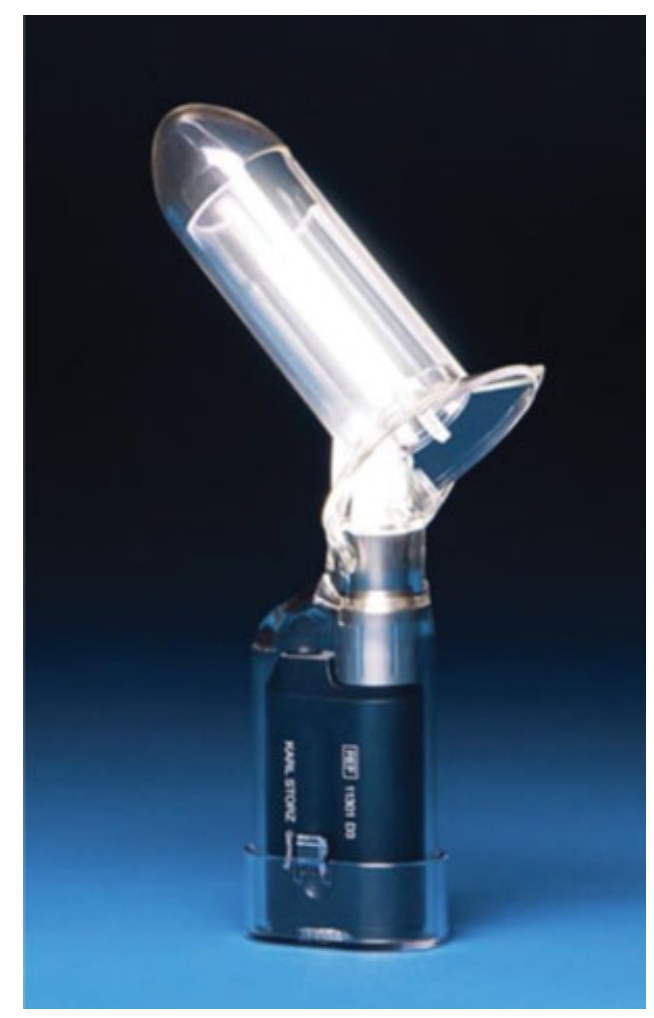

Fig. 1 EndoPex AC002 Anuscope. 

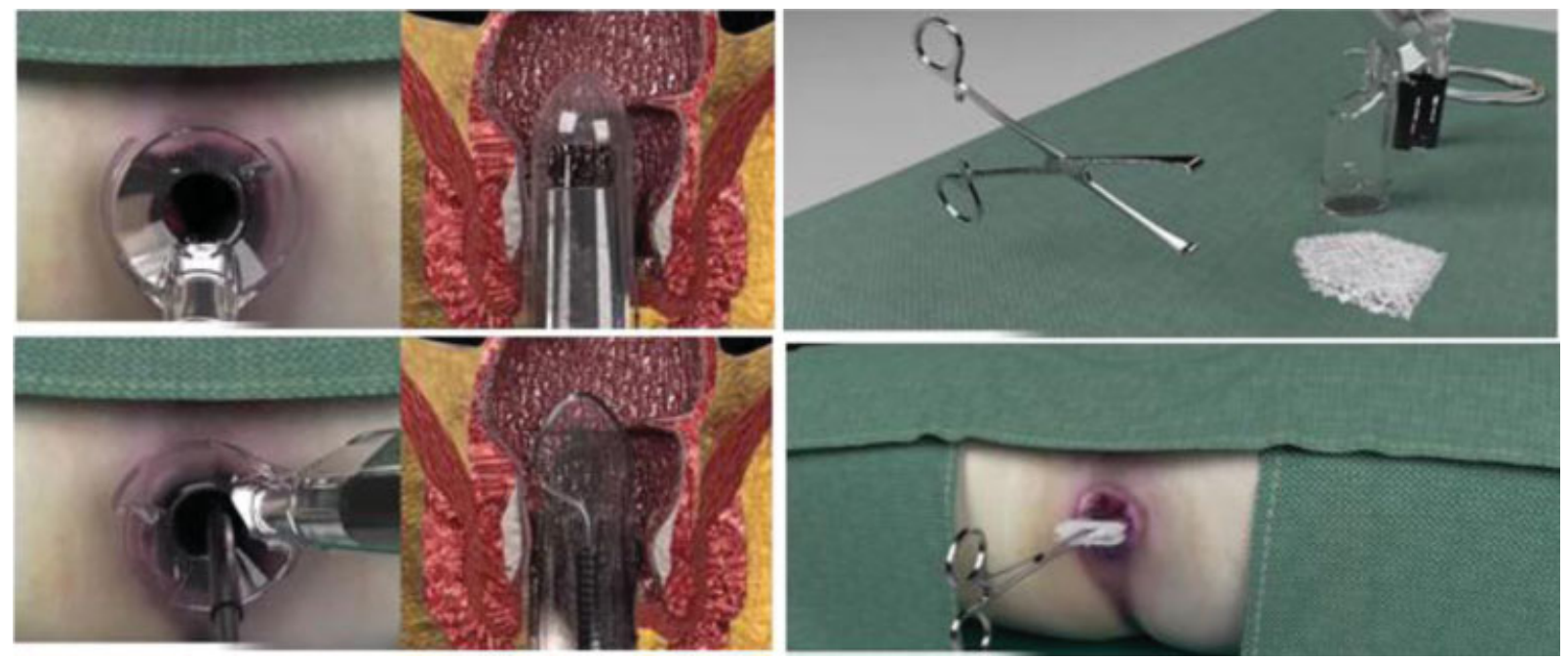

Fig. 2 and 3 Using 3 gauze, an envelope is created to evidence symptomatic hemorrhoids (ROTTA maneuver). The lubricated anuscope is introduced into the anal canal to the nipples detected as sick and the best sound of arterial flow is sought with the doppler probe.

SHeLF. Despite the use of different anoscopes, all of them were produced to perform hemorrhoidal dearterialization and all have doppler.

\section{Surgical technique "Selective hemorrhoidal dearterialization with high mucopexy - SHeLF"}

All patients were submitted to mechanical preparation of the rectus with clyster containing $118 \mathrm{ml}$ of dibasic sodium phosphate $0.06 \mathrm{~g} / \mathrm{ml}$ and monobasic sodium phosphate $0.16 \mathrm{~g} / \mathrm{ml}$ (Fleet-enema ${ }^{\rightarrow}$ ), ${ }^{1}$ applied from 9 to 10 hours before the procedure. Surgical interventions were performed under spinal anesthesia. All patients received antibiotic prophylaxis with $\mathrm{Kefazol}^{2}(500 \mathrm{mg}$ intravenously during anesthetic induction) repeated at discharge (between 7 and 10 hours after surgery). All interventions were performed with the patients in the lithotomy position.

In the SHeLF technique, before rectal touch, three dry gauze are introduced, folded and fastened in Allis tweezers, tossing them out, thus bringing the altered cushions out of the anal canal, which enables their visualization (we call it the rotta maneuver - - Figure 3). It is then defined which hemorrhoidal nipples will be submitted to selective arterial ligation and mucopexy. Then, gentle anal dilation is made with ultrasound gel and the index finger, after lubricating the uniscope with the same gel, and carefully introducing it until it reaches the lower part of the rehest, $\sim 7$ centimeters above the anal edge, directing the working window to one of the prolapsed cushions (-Figure 2 ). The transducer is then introduced, touching the exposed region of the distal rehest mucosa above the cushion. It slides in the craniocaudal and lateral-lateral direction until the highest intensity of the acoustic signal generated from the arterial branch identified in the rectum wall is heard. Thus, with the arterial branch feeder of the cushion located, a needle carrier is introduced

\footnotetext{
1 Fleet Laboratories, Lynchburg, Virginia, USA.

2 Sanofi-Aventis Farmacêutica Ltda., Suzano, São Paulo, Brazil.
}

by the special uniscope mounted with a cylindrical curved needle, with $5 / 8$ circumference, $2.6 \mathrm{~cm}$ long, already coupled to the absorbable monofilament wire (2-0), supplied in conjunction with the system. Dearterialization is initiated at the chosen location, applying two transfixing points in "X", manually tied (-Figure 4). After ligation, 2 more points (simple stitching) are placed apart from each other - at a distance between 1.0 and $1.5 \mathrm{~cm}$ - cranially and manually tied to the transfixing point of dearterialization ( - Figure 5 ). The distal points (simple stitching) are started - between 1.0 and $1.5 \mathrm{~cm}$ - for the correction of the mucous prolapse, which is corrected by continuous suture (three to four strides) performed under direct vision in the direction of the retane to the anal canal (-Figure 6 ) and is facilitated by the rotation of the mobile part of the endoscope, thus expanding the work area. The suture progresses up to 0.5 to $1.0 \mathrm{~cm}$ above the pectin line. Mucopexy is completed by tying the last point passed to the transfixing of the dearterialization. The procedure is repeated for the other altered cushions.

After the procedure, patients were discharged with a prescription of dipyrone $(500 \mathrm{mg})$ every six hours; ketoprofen $(1,000 \mathrm{mg} /$ day); and $2 \%$ topical use lidocaine and Tramadol hydrochloride with paracetamol $(200 \mathrm{mg} /$ day $)$ in case of severe pain. Patients were also instructed to perform seat baths with warm water after evacuations. The first postoperative return is made on the $5^{\text {th }}$ postoperative day and, afterwards, on the $4^{\text {th }}$ week. If at the first visit the patient complains of more severe pain or any other symptom, outpatient follow-up becomes weekly and, afterwards, monthly.

\section{Findings}

The mean duration of the surgical procedure was $20 \pm 10$ minutes to identify the altered cushions and perform dearterialization and mucopexy. Only where there were altered cushions, dearterialization points with high mucopexy were applied. In addition to dearterialization and mucopexy, there 

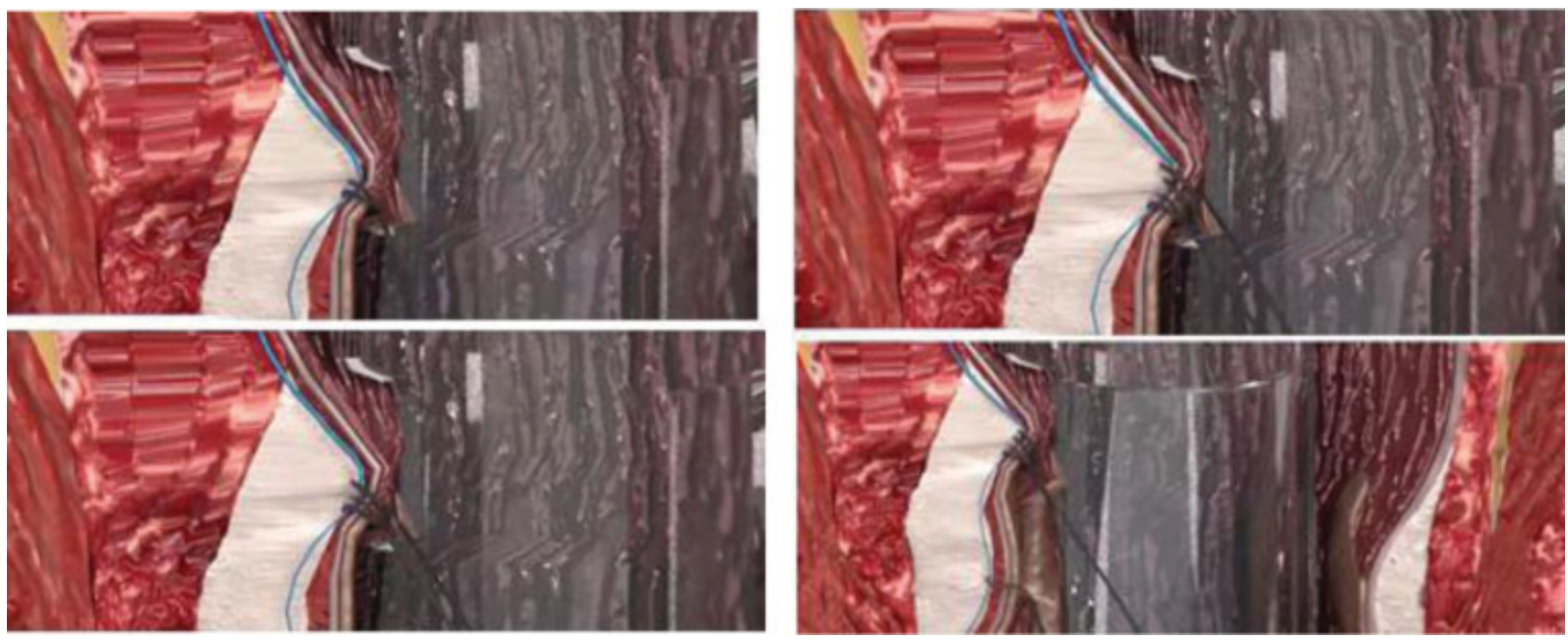

Fig. 4 and 5 Arterial ligation with point in "X" and high pexia with 2 cranial points. Node and beginning of pexia or traditional lifting (SHeLF).
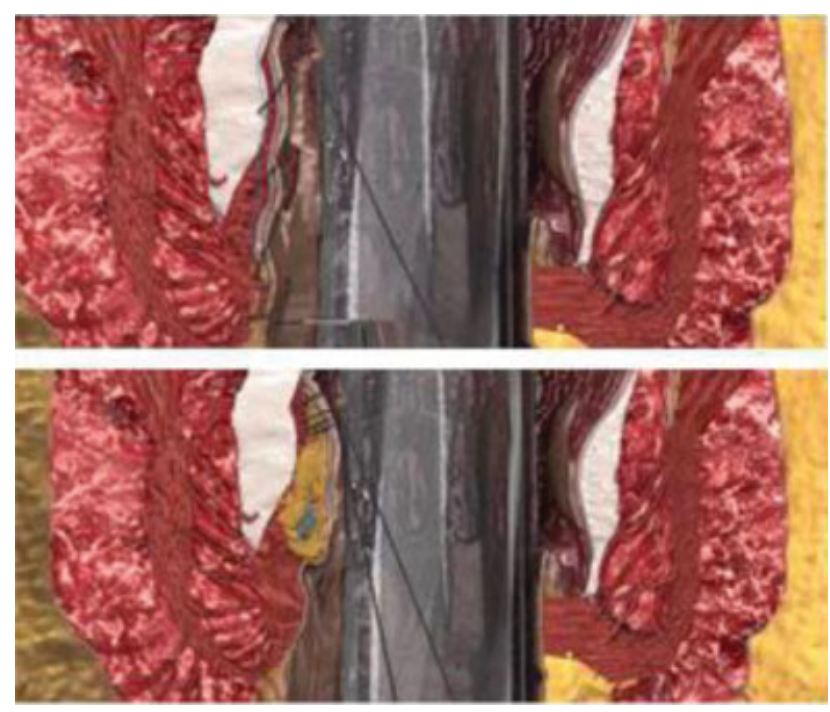

Fig. $63-4$ passed from 1 to $1.5 \mathrm{~cm}$ away, finishing $0.5 \mathrm{~cm}$ from the pectinline.

was a need for resection (one or more) of untolerated plicomas.

-Table 2, 3, 4 and 5 show the main postoperative complaints comparing the two techniques. Pain was a postoperative complaint reported by 13 (11.82\%) patients undergoing DGHD + M and by 19 (10.44\%) subjected to SHeLF. Intense

Table 2 Main postoperative complaints

\begin{tabular}{|l|l|l|l|}
\hline Technique & DGHD $+\mathrm{M}$ & SHeLF & Significance $^{*}$ \\
\hline Pain & $\mathbf{1 1 . 8 \%}$ & $10.4 \%$ & $\mathbf{p}<\mathbf{0 . 0 3}$ \\
\hline Intense tenesmo & $23.6 \%$ & $3.8 \%$ & $\mathbf{p}<\mathbf{0 . 0 0 0 0 0 0 0 2}$ \\
\hline Bleeding & $2.7 \%$ & $0.5 \%$ & $p<0.001$ \\
\hline Thrombosis & $5.5 \%$ & $0.5 \%$ & $p<0.000006$ \\
\hline Relapse & $5.5 \%$ & $5.0 \%$ & $p<0.4$ \\
\hline
\end{tabular}

Abbreviations: DGHD + M: Doppler-Guided Hemorrhoidal Dearterialization + craniocaudal mucopexy by manual suture, SheLF, selective hemorrhoidal high lifting.

*Pearson chi-squared test. tenesmus occurred in $26(23.64 \%)$ patients submitted to $\mathrm{DGHD}+\mathrm{M}$ and in 7 (3.85\%) subjected to SHeLF. Three patients $(2.73 \%)$ submitted to $\mathrm{DGHD}+\mathrm{M}$ and 1 patient (0.55\%) submitted to SHeLF presented with postoperative bleeding, and $1(0.55 \%)$ patient in the DGHD + M group required surgical hemostasis. Six patients (5.45\%) who underwent DGHD + M and 8 (4.39\%) submitted to the SHeLF technique were reoperated for recurrence.

Table 3 Number of hemorrhoidal nipples operated per patient

\begin{tabular}{|l|l|l|l|l|}
\hline $\begin{array}{l}\text { NUMBER OF } \\
\text { OPERATED } \\
\text { NIPPLES }\end{array}$ & \multicolumn{2}{|l|}{ DGHD +M } & \multicolumn{2}{l|}{ SHeLF } \\
\hline $\mathbf{1}$ & 0 & - & 4 & $2.20 \%$ \\
\hline $\mathbf{2}$ & 0 & - & 30 & $16.48 \%$ \\
\hline $\mathbf{3}$ & 0 & - & 80 & 43.96 \\
\hline 4 & 0 & - & 58 & 31.87 \\
\hline $\mathbf{5}$ & 0 & - & 10 & $5.49 \%$ \\
\hline 6 & 110 & $100 \%$ & 0 & - \\
\hline
\end{tabular}

Abbreviations: DGHD + M: Doppler-Guided Hemorrhoidal Dearterialization + craniocaudal mucopexy by manual suture, SheLF, selective hemorrhoidal high lifting.

Table 4 Location of nipples operated by the SHeLF technique

\begin{tabular}{|l|l|l|}
\hline $\begin{array}{l}\text { OPERATED NIPPLES } \\
\text { Hours }\end{array}$ & \multicolumn{2}{l|}{ SHeLF } \\
\hline $3,7,11$ & 59 & $32.41 \%$ \\
\hline $3,5,7,11$ & 46 & $25.27 \%$ \\
\hline $5,7,11$ & 9 & $4.94 \%$ \\
\hline $3,5,7$ & 6 & $3.29 \%$ \\
\hline 5,11 & 6 & $3.29 \%$ \\
\hline 3,7 & 5 & $2.74 \%$ \\
\hline
\end{tabular}

Abbreviation: SheLF, selective hemorrhoidal high lifting. 
Table 5 Location of nipples operated in surgery with SHeLF

\begin{tabular}{|l|l|l|}
\hline $\begin{array}{l}\text { Position of operated nipples } \\
\text { (hours) }\end{array}$ & \multicolumn{2}{|l|}{ SHeLF } \\
\hline 1 & 11 & 6.04 \\
\hline 3 & 146 & 80.21 \\
\hline 5 & 97 & 53.29 \\
\hline 7 & 156 & 85.71 \\
\hline $\mathbf{9}$ & 16 & 8.79 \\
\hline 11 & 145 & 79.67 \\
\hline
\end{tabular}

Abbreviation: SheLF, selective hemorrhoidal high lifting.

\section{Discussion}

The best surgical option for the treatment of symptomatic HD has as main requirements: to provide remission of symptoms, simple execution, affordable cost, being well tolerated, and to present low rates of complications and recurrence. ${ }^{14,15}$ The large number of surgical procedures currently in use for HD treatment shows that none of the proposed techniques could unite all these predicates. Excisional hemorrhoidectomy techniques are currently the most widely used surgical option worldwide. Although effective in controlling symptoms and evolving with low recurrence rates, they present as limitations the intensity of postoperative pain and care during convalescence that keep the patient away from activities for a considerable period of time. ${ }^{14,15}$ In order to develop alternative methods capable of minimizing the inconveniences of excisional hemorrhoidectomy, new options have emerged for the treatment of $\mathrm{HD}^{8,11}$ The DGHD $+\mathrm{M}$ technique represents one of the surgical options used..$^{8,16-19}$ Despite the advantages of the method regarding the reduction of postoperative pain and faster return of the patient to social life, some symptoms related to the greater number of sutures performed in the DGHD $+\mathrm{M}$ technique, often unnecessary, and the highest rates of recurrence of the disease, are still outcomes that satisfy the doctor and the patient. ${ }^{19}$ A recent study using the DGHD $+\mathrm{M}$ technique that evaluated 1,000 patients showed recurrence rates in HD grades II, III and IV of $8.5,8.7$, and $18.1 \%$, respectively; ${ }^{20}$ $3.1 \%$ of patients had tenesmus and $7 \%$ had surgery for recurrence. Other authors have shown that the use of DGHD $+\mathrm{M}$ in patients with grade III HD, when compared with ligation and mucopexy isolated from the hemorrhoidal cushions, presented considerably longer surgical time (31 minutes versus. 9 minutes), higher intensity of postoperative pain on the visual analog scale (4.4 versus 2.2 ), higher analgesic consumption and for a longer period (17 tablets versus 11 tablets for 13 days versus 9 days), with the same recurrence rates. ${ }^{21}$

A Russian study using DGHD $+\mathrm{M}$ interrupted blood flow in six arterial branches. ${ }^{22}$ It was found that the mean surgical time was $33 \pm 12$ minutes. In the postoperative follow-up, 3 patients (8.6\%) presented external hemorrhoidal thrombosis and 1 required additional surgical treatment. Two (5,7\%) patients presented with postoperative bleeding, and surgical hemostasis was required in $1 .^{22}$ Five (14.3\%) presented with urinary retention that required bladder probing. It is worth noting that when compared with the results of the present study, these numbers were much higher. In a mean postoperative follow-up of 10 months (between 2 and 28 months), the author showed that there was a significant improvement in symptoms in 33 (94\%) patients. Nine (25,7\%) patients evolved with irregular bleeding in bowel movements in the first few weeks, which resolved spontaneously. Three (8,6\%) patients had mild anal pain, $4(11.4 \%)$ transient anal ardor, and $4(11.4 \%)$ tenesmuses. In the late follow-up, it was found that $10(28.6 \%)$ patients evolved with some degree of prolapse, and $2(5.7 \%)$ prolapses were more significant, requiring surgical correction. The author did not find any case of susthesis or of anal incontinence. In the satisfaction questionnaire, there was a significant number of patients satisfied with the method. ${ }^{22}$ Although it suggests that the DGHD +M technique can be applied to patients with grade IV HD, its postoperative results, as with AM, do not present the same degree of satisfaction when compared with patients with less advanced HD. ${ }^{21}$

A second multicenter study evaluating 507 HD patients with grade II (28.4\%), grade III (63\%), and grade IV (8.6\%) HD submitted to DGHD + M who were followed-up for 1 year found good results in $69.2 \%$ of the patients and acceptable results in the remaining $4.8 \%{ }^{16}$ When patients were grouped according to the HD grade, the study showed that $92.4 \%$ of HD patients with grade II and $84 \%$ with grade III were satisfied, compared with $41 \%$ of $\mathrm{HD}$ patients classified as grade IV. ${ }^{16}$

A systematic review of the literature evaluating DGHD + $\mathrm{M}$, with 2,000 patients showed that $9.7 \%$ complained of postoperative bleeding, $8.7 \%$ complained of pain during bowel movements, ${ }^{17}$ and $10.8 \%$ presented recurrence of mucous prolapse and, when considering only patients followed-up for $>1$ year, prolapse and bleeding recurrence increased to 10.8 and $9.7 \%$, respectively. ${ }^{17}$

Ferrandis et al., in 2020, evaluated 150 patients submitted to DGHD + M who were followed-up for 60 months. Fifty (33.3\%) patients were lost to follow-up, 100 patients remained (47 female, 53 male), and there were no serious complications in 5 years. Bleeding, local discomfort, and pain scores were improved. There was recurrence in 36 patients (35.6\%), 20 (19.8\%) of which were submitted to reoperation. They concluded that there was low invasivity, low morbidity and satisfactory functional results and that, despite the long-term recurrence rate, only $19.8 \%$ needed a second surgery. None of the patients presented acute urinary retention, surgical site infection, anal snose, or incontinence. The studied symptoms (pain, hemorrhoidal prolapse, bleeding and local discomfort) improved immediately after surgery. ${ }^{18}$

When analyzing the objectives of the DGHD $+\mathrm{M}$ and SHeLF techniques, the two procedures were designed with the objective of curing HD considering the two main etiopathogenic factors: blood hyperflow and mucous prolapse. Both procedures disrupt arterial blood flow to the hemorrhoidal venous plexus and fix the excess of prolapsed rectal mucosa, resessecting the plicoma when necessary. ${ }^{22}$ The 
SHeLF technique was conceived based on the therapeutic proposal of the DGHD + M technique, also interrupting blood flow by applying transfixing points on the arterial branches and subsequent fixation of hemorrhoidal cushions prolapsed, but in a higher situation when compared to the classical procedure. ${ }^{1}$ Another difference in relation to the standardized technique of DGHD $+\mathrm{M}$ is that the application of the point for ligation in the arterial branch identified by doppler and mucopexy is only performed in the cushions of the patient. In these cushions, mucopexy is in a higher position (farther from the pectin line). Mucopexy is performed by a simple suture, with two stitches, above the traditional "X" stitch performed during dearterialization. These mucopexy points are manually tied to the point of dearterialization. After this ligation is performed at the highest point of the rectal mucosa, the continuous suture towards the pectinline begins. Rectal mucopexy in the SHeLF technique is always performed in the longitudinal direction of the restum, remaining free spaces between each suture. With the suture, less venous flow block is obtained by the hemorrhoidal plexus, which is probably responsible for the cases of postoperative hemorrhoidal thrombosis that occur in the DGHD + M technique. The greater interval between the stitches also reduces the possibility of sthesis and the sensation of decreased light of the retitus.

We ourselves, evaluating 407 patients with HD grades II (6.6\%), III (59\%) and IV (28.8\%) submitted to DGHD + M, found that tenesmus was the main complaint, reported by $93.36 \%$ of the patients. ${ }^{12}$ In 43 (10.5\%) of the 379,98 patients presented intense tenesmo and $44(10.81 \% \%)$ patients presented from moderate to intense tenesmo, 4 patients had major postoperative bleeding, and $1(0.5 \%)$ of them required revision of hemostasis and blood replacement. In the postoperative follow-up, 18 (4.42\%) patients evolved with prolapse, 11 (2.7\%) with thrombosis of $\geq 1$ hemorrhoidal cushions, and 19 (4.6\%) patients required resurgery for HD relapse.

In view of these high rates of tenesmus, we started to use the SHeLF technique to treat grades II, III and IV HD patients in order to verify whether this new strategy could reduce the incidence of tenesmus and of hemorrhoidal thrombosis in relation to DGHD + M. Despite the small number of patients included in the present study and the short postoperative follow-up time, the results found that the SHeLF technique can be considered a valid option for the treatment of HD. We found that, with the SHeLF technique, there was a significant reduction in surgical time, tenesmus, urinary retention, and postoperative bleeding. However, in relation to postoperative pain, the SHeLF technique showed no difference in relation to DGHD $+\mathrm{M}$.

The results of the present study show that SHeLF is a simple surgical procedure with a short learning curve due to the need to repeat the procedure several times in the same patient. The technique provides improvement of symptoms, especially in patients with grades II and III HD, besides preseting acceptable rates of postoperative complications and short-term recurrence. There were no serious complications. A national multicenter study, including a larger number of patients and longer follow-up period, is essential to confirm the validity of this new option for HD treatment. Only in this way will it be possible to confirm the preliminary results of the present study.

\section{Conclusion}

The preliminary results found with the technical use of SHeLF for the surgical treatment of grades II, III, and IV HD were promising, having as main benefits the reduction of pain $(p<0.03)$ and of postoperative tenesmus $(p<0.00000002)$, maintaining the recurrence rate of SHeLF $(p<0.4)$ when compared with the DGHD + M technique.

\section{Source of funding}

None.

\section{Conflict of interests}

Rotta C.M. developed the SHeLF technique.

\section{References}

1 Rotta, Carlos Mateus, et al. Doppler-guided hemorrhoidal artery ligation with rectal mucopexy technique: initial evaluation of 42 cases. Journal of Coloproctology (Rio de Janeiro) [online]. 2012;32 (04)

2 Kaidar-Person O, Person B, Wexner SD. Hemorrhoidal disease: A comprehensive review. J Am Coll Surg 2007;204(01):102-117. Doi: 10.1016/j.jamcollsurg.2006.08.022

3 Johanson JF, Sonnenberg A. The prevalence of hemorrhoids and chronic constipation. An epidemiologic study. Gastroenterology 1990;98(02):380-386. Doi: 0.1016/0016-5085(90)90828-o

4 Altomare DF, Roveran A, Pecorella G, Gaj F, Stortini E. The treatment of hemorrhoids: guidelines of the Italian Society of Colorectal Surgery. Tech Coloproctol 2006;10(03):181-186. Doi: 10.1007/s10151-006-0277-y

5 Cataldo P, Ellis CN, Gregorcyk S, et al; Standards Practice Task Force, The American Society of Colon and Rectal Surgeons, USA. Practice parameters for the management of hemorrhoids (revised). Dis Colon Rectum 2005;48(02):189-194. Doi: 10.1007/ s10350-004-0921-4

6 Milligan ET, Morgan CN, Jones LE, et al. Surgical anatomy of the anal canal and the operative treatment of haemorrhoids. Lancet 1937;230:1119-1124

7 Ferguson JA, Heaton JR. Closed hemorrhoidectomy. Dis Colon Rectum 1959;2(02):176-179. Doi: 10.1007/bf02616713

8 Longo A. Treatment of hemorrhoidal disease by reduction of mucosa and hemorrhoidal prolapse with a circular suturing device: a new procedure. In: Proceedings of the sixth world congress of endoscopic surgery,. Bologna, Italy.: Monduzzi Publishing Co.; 1998:777-784

9 Bellio G, Pasquali A, Schiano di Visconte M. Stapled hemorrhoidopexy: Results at 10-year follow-up. Dis Colon Rectum 2018;61 (04):491-498. Doi: 10.1097/DCR.0000000000001025

10 Davis BR, Lee-Kong SA, Migaly J, Feingold DL, Steele SR. The American Society of Colon and Rectal Surgeons clinical practice guidelines for the management of hemorrhoids. Dis Colon Rectum 2018;61(03):284-292. Doi: 10.1097/DCR.0000000000001030

11 Morinaga K, Hasuda K, Ikeda T. A novel therapy for internal hemorrhoids: ligation of the hemorrhoidal artery with a newly devised instrument (Moricorn) in conjunction with a Doppler flowmeter. Am J Gastroenterol 1995;90(04):610-613

12 Rotta CM, Machado LR, Uwada PM, et al. Postoperative results of 407 patients submitted to Doppler-guided transanal hemorrhoidal dearterialization. J Coloproct (Rio J.) 2019;39(03):211-216. Doi: $10.1016 /$ j.jcol.2019.04.001 
13 Goligher JC Ed. Surgery of the anus, rectum and colon. 5. Aufl age. W. B. Saunders; 1983

14 Giordano P, Nastro P, Davies A, Gravante G. Prospective evaluation of stapled haemorrhoidopexy versus transanal haemorrhoidal dearterialisation for stage II and III haemorrhoids: three-year outcomes. Tech Coloproctol 2011;15(01):67-73. Doi: 10.1007| s10151-010-0667-z

15 Lienert M, Ulrich B. [Doppler-guided ligation of the hemorrhoidal arteries. Report of experiences with 248 patients]. Dtsch Med Wochenschr 2004;129(17):947-950. Doi: 10.1055/s-2004-823061

16 Ratto C, Giordano P, Donisi L, Parello A, Litta F, Doglietto GB. Transanal haemorrhoidal dearterialization (THD) for selected fourth-degree haemorrhoids. Tech Coloproctol 2011;15(02): 191-197. Doi: 10.1007/s10151-011-0689-1

17 Cantero R, Balibrea JM, Ferrigni C, et al. [Doppler-guided transanal haemorrhoidal dearterialisation. An alternative treatment for haemorrhoids]. Cir Esp 2008;83(05):252-255. Doi: 10.1016/ s0009-739x(08)70563-3

18 Ferrandis C, De Faucal D, Fabreguette JM, Borie F. Efficacy of Doppler-guided hemorrhoidal artery ligation with mucopexy, in the short and long terms for patients with hemorrhoidal disease. Tech Coloproctol 2020;24(02):165-171. Doi: 10.1007/s10151019-02136-1

19 Ratto C, Campennì P, Papeo F, Donisi L, Litta F, Parello A. Transanal hemorrhoidal dearterialization (THD) for hemorrhoidal disease: a single-center study on 1000 consecutive cases and a review of the literature. Tech Coloproctol 2017;21(12):953-962. Doi: 10.1007/ s10151-017-1726-5

20 Gupta PJ, Kalaskar S, Taori S, Heda PS. Doppler-guided hemorrhoidal artery ligation does not offer any advantage over suture ligation of grade 3 symptomatic hemorrhoids. Tech Coloproctol 2011;15(04):439-444. Doi: 10.1007/s10151-011-0780-7

21 Zagriadskiî EA. [Trans-anal disarterization of internal hemorrhoids under Doppler control with mucopexy and lifting in treatment of stage III-IV hemorrhoids]. Khirurgiia (Mosk) 2009; 2(02):52-58

22 Pol RA, van der Zwet WC, Hoornenborg D, et al. Results of 244 consecutive patients with hemorrhoids treated with Dopplerguided hemorrhoidal artery ligation. Dig Surg 2010;27(04): 279-284. Doi: 10.1159/000280020 